\section{Augmented vertical recti transposition with intraoperative botulinum toxin for complete and chronic sixth nerve palsy}

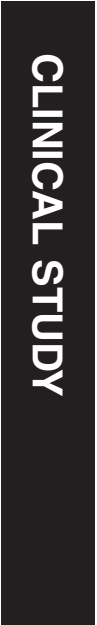

Department of

Ophthalmology, Nikookari

Eye Center, Tabriz University of Medical sciences, Tabriz, Iran

Correspondence: R Nabie, Department of Ophthalmology, Nikookari Eye Center, Abbasi Street, Tabriz 5154645395, Iran Tel: +98 41 36551332; Fax: +984136577336.

E-mail: r_nabie@yahoo.com

Received: 28 March 2016 Accepted in revised form: 14 September 2016 Published online: 4 November 2016

\begin{abstract}
Purpose To evaluate the results of augmented vertical rectus muscle transposition (VRT) with intraoperative botulinum toxin (BTX) for complete and chronic sixth nerve palsy.

Methods During a 10-year period (20042014) all patients with chronic and complete sixth nerve palsy and contracted medial rectus (MR) who underwent augmented VRT and BTX injection into the MR enrolled in this study.

Results In total, 29 patients (5 bilateral) were enrolled in this study. Preoperative deviation was $45 \pm 17.5$ Prism Diopter (PD), which was improved to $-3.1 \pm 13.2$ after the operation $(P<0.001)$. Mean preoperative and postoperative abduction limitation was $-4.4 \pm 1.1$ and $-1.8 \pm 0.9$, respectively $(P<0.001)$. The success rate was $76 \%$ (deviation within 10 PD of orthotropia). Four patients (13.7\%) had hypotropia. In 19 patients with preoperative deviation $\leq 45 \mathrm{PD}$, four patients had consecutive exotropia. Conclusion Intraoperative BTX injection with augmented vertical rectus transposition is an effective procedure. In deviation $\leq 45$ there is a risk of overcorrection. Eye (2017) 31, 148-151; doi:10.1038/eye.2016.226; published online 4 November 2016
\end{abstract}

\section{Introduction}

Patients with sixth nerve palsy present limitation of abduction, moderate-to-severe esotropia and diplopia in primary position, and usually abnormal head posture. ${ }^{1}$ Various surgical procedures have been described the management of complete sixth nerve palsy, including partial and full vertical recti muscles transposition (VRT) to induce the abduction of
R Nabie and D Andalib

the eye. Full VRT is among the most effective treatment for the management of the complete sixth nerve palsy. ${ }^{2}$ Foster-introduced augmented VRT to enhancing abduction. In long-standing palsy, the medial rectus muscle (MR) is contractured and weakening of the MR may be required. Simultaneous operation on MR has the risk of anterior segment ischemia. ${ }^{3}$ To avoid this complication, some authors advocated botulinum toxin (BTX) injection to the MR. ${ }^{4-7}$

The use of intraoperative BTX to weaken the antagonist MR in transposition procedures has been proposed by Scott. ${ }^{8}$ Scott and Kraft postulated that BTX reduces contracture of the MR and allows for more complete recovery of LR muscle function. ${ }^{9}$

In this study, we describe the results of augmented transposition of vertical rectus muscles with intraoperative BTA in the management of chronic sixth nerve palsy.

\section{Methods}

The Medical Ethics Committee of Tabriz University of Medical Sciences approved this study, and informed consent was obtained from patients. During a 10-year period between 2004 and 2014, patients with complete and chronic sixth nerve palsy enrolled in this study. We defined complete palsy as deficient abduction beyond midline and chronic at least 6 months from the onset of palsy. Patients included in the analysis had at least 6 months follow-up. The senior author (RN) performed all surgeries.

The etiology of the palsy was classified at the time of enrollment as traumatic, congenital, neoplastic, vascular, and idiopathic. Abduction deficit was graded from -8 (globe immobilized in extreme adduction) to -4 (abduction to midline) to 0 (full abduction). A forced muscle 
generation test confirmed the presence of complete sixth nerve palsy and forced duction testing confirmed the MR contracture. Patients must have MR contracture to be included in the study. The following data were collected and analyzed: age at enrollment, etiology, gender, involved eye, pre- and postoperative deviation, and abduction deficit and duration of follow-up. Patients with history of previous surgery or BTX injection and visual acuity of $<20 / 200$ were excluded. Angle of deviation was measured by simultaneous prism and cover tests in the primary position at distance fixation. Augmented vertical rectus muscles transposition was performed according to the technique described by Foster. ${ }^{2}$ In previous studies, 2.5-5 IU of Botox was injected to MR. ${ }^{4-7}$ After the completion of transposition, 12.5 IU of BTX A (Dysport, Ipsen, Berkshire, UK) were injected into MR muscle (3 units of Dysport is equivalent to 1 unit of Botox). ${ }^{8}$ Chloramphenicol eye drops were prescribed for 1 week and betamethason eye drops were prescribed for 2 weeks. Motility and complete eye examination, including signs and symptoms of anterior segment ischemia, were performed regularly. The SPSS 17 and the KolmogorovSmirnov test were used for descriptive data, and pre-and postoperative results were analyzed by the Wilcoxon signed-rank test. $P$-values of $\leq 0.5$ were considered significant. Postoperative alignment within 10 prism diopters (PD) of orthotropia was defined as success.

\section{Results}

In total, 29 patients (24 unilateral and 5 bilateral) with sixth nerve palsy enrolled in the study, of which 15 of the patients were male (51.71) and 14 (49.3\%) were female. Etiology and laterality are summarized in Table 1 . The most common cause of sixth nerve palsy in this study was trauma (48.1\%), followed by congenital causes (31\%). The pre- and postoperative data are summarized in Table 2.

The mean age was $26.5 \pm 19.8$ years (range $1-62$ years). Follow-up period was $24.2 \pm 24.3$ months (range 6-91 months). Twenty-two (76\%) patients were within 10 PD of orthophoria, and the rates of undercorrection and overcorrection were $3(10.3 \%)$ and 4 (13.7\%), respectively. The mean preoperative deviation was $45 \mathrm{PD}$ that reduced

Table 1 Etiology, laterality and involved eye

\begin{tabular}{lcccc}
\hline & Unilateral & Bilateral & OD & OS \\
\hline Trauma & 12 & 2 & 8 & 8 \\
Congenital & 8 & 1 & 4 & 6 \\
Neoplasia & 1 & - & - & 1 \\
Vascular & 1 & - & - & 1 \\
Convulsion & - & 2 & 2 & 2 \\
Unknown & 2 & - & - & 2 \\
\hline
\end{tabular}

to -3 (exotropia shift) postoperatively $(P<0.001)$. The effect of operation was 48.1 \pm 17.7 PD (range 12-90). In 3 patients with under correction, preoperative ET were 90, 60 and 30 PD, and postoperative ET were 30, 15, and 18, respectively. In overcorrected patients, preoperative ET were $45,40,25$, and $20 \mathrm{PD}$ with postoperative results of $18,20,40$, and 35 PD of XT in primary position. In 19 patients, preoperative ET were $\leq 45$, in which 4 patients (21\%) had more than 10 PD XT after operation. Two of these 4 patients had limited adduction of -1 and -2 . In 5 patients $(16.9 \%)$, we observed postoperative limitation of adduction ranging from -1 to -2 . All of the patients with consecutive XT underwent reoperation and achieved orthotropia. In three patients, we performed unilateral or bilateral MR resection and in one patient LR recession and $\mathrm{MR}$ resection. In two patients with consecutive $\mathrm{XT}$ and limitation of adduction, MR resection resulted in full adduction. None of these 4 patients developed anterior segment ischemia. Four patients $(13.7 \%)$ had postoperative hypotropia of 30, 20, 10, and $8 \mathrm{PD}$. The patient with 30 PD hypotropia underwent 2 operations on transposed IR that diminished hypotropia to $10 \mathrm{PD}$. The other 3 patients with hypotropia refused reoperation.

Preoperation abduction limitation was $-4.4 \pm 1.1$ (range -4 to -8 ), and that improved to $-1.8 \pm 0.9$ (range 0 to -4$)$, which was statistically significant $(P<0.001)$.

We compared congenital and traumatic cases. The results were marginally different between the 2 groups and this difference was not statistically different (Table 3 ).

\section{Discussion}

In complete sixth nerve palsy, when the globe cannot be excursed beyond midline, transposition procedure is indicated. Various transposition procedures have been described for management of sixth nerve palsy. The goals of surgery are to improve eye alignment in primary position, increase the binocular diplopia-free field and improve abduction deficit. Full-tendon VRT was first described by Schillinger ${ }^{10}$ in 1959. In 1997, Foster ${ }^{2}$ introduced augmented VTR to enhance abducting force. MRI evaluation of this technique shows increased lateralization of the transposed muscles. In chronic sixth palsy, passive abduction may be limited secondary to MR contracture.

To improve abduction, MR contracture must be alleviated. Considering the risk of anterior segment ischemia with simultaneous MR recession, we used intraoperative BTX injection of MR.

In our study, the most common cause of palsy was trauma, which was compatible with other studies. ${ }^{4,11}$ Our success rate was $76 \%$. Armenia, ${ }^{7}$ Fitzsimons, ${ }^{5}$ and Leiba et $a l^{12}$ performed unaugmented VRT with intraoperative 
Table 2 Pre-and postoperative data

\begin{tabular}{|c|c|c|c|c|c|c|}
\hline \multirow{2}{*}{$\frac{\text { Age }}{(26.5 \pm 19.8)}$} & \multicolumn{2}{|c|}{ Deviation } & \multicolumn{2}{|c|}{ LR underaction } & \multicolumn{2}{|c|}{ Postop MR underaction FU } \\
\hline & Preop & Postop & Preop & Postop & $5(16.9 \%)$ & - \\
\hline & $\begin{array}{l}45 \pm 17.5 \\
(20 \text { to } 90)\end{array}$ & $\begin{array}{c}-3.1 \pm 13.2^{\mathrm{a}} \\
-\end{array}$ & $\begin{array}{l}-4.4 \pm 1.1 \\
(-8 \text { to }-4)\end{array}$ & $\begin{array}{l}-1.8 \pm 0.9 \\
(0 \text { to }-4)\end{array}$ & $\begin{array}{c}-0.2 \pm 0.4 \\
\text { (range, }-1 \text { to }-2 \text { ) }\end{array}$ & $\begin{array}{l}24.2 \pm 24.3 \\
(6 \text { to } 91 \mathrm{mo})\end{array}$ \\
\hline Effect: & $48.1 \pm 17.7(12-90)$ & & & & & \\
\hline Success: & $22(76 \%)$ & & & & & \\
\hline Undercorrection: & $3(10.3 \%)$ & & & & & \\
\hline Overcorrection & $4(13.7 \%)$ & & & & & \\
\hline
\end{tabular}

${ }^{a}$ Negative post-operative deviation referred to consecutive exotropia.

Table 3 Comparing traumatic and congenital 6th nerve palsy

\begin{tabular}{lccc}
\hline & Traumatic & Congenial & P-value $^{\mathrm{a}}$ \\
\hline Preop deviation & $48.3 \pm 18.5$ & $45 \pm 21.9$ & 0.48 \\
Postop deviation & $-0.67 \pm 11.20^{\mathrm{b}}$ & $-2.66 \pm 15.3$ & 0.95 \\
Preop LR underaction & $-4.8 \pm 1.5$ & $-4.1 \pm 0.3$ & 0.35 \\
Postop LR underaction & $-1.7 \pm 0.9$ & $-1.6 \pm 1.1$ & 0.82 \\
Postop adduction & $-0.06 \pm 0.2$ & $-0.1 \pm 0.3$ & 0.86 \\
deficiency & & & \\
\hline
\end{tabular}

${ }^{\mathrm{a}}$ Mann-Whitney Test. ${ }^{\mathrm{b}}$ Negative postoperative deviation referred to consecutive exotropia.

BTA and gained 60,50 , and $59 \%$ success rates, respectively.

In the present study, the mean final reduction in esotropic deviation was $48 \mathrm{PD}$. In previous studies that used unaugmented VRT with BTX, exotropic shift was between 32 and $66 \mathrm{PD}$, however, some authors have included patients with previous surgery. ${ }^{4-7,12,13}$ In the current study, mean abduction deficit improved from -4.4 to -1.8 , which seems to be slightly better than most of previously-mentioned transposition procedures. ${ }^{47,11}$ Four $(13.7 \%)$ of our patients were overcorrected. In all these patients, preoperation esotropia was $\leq 45$. We assessed LR rectus function by office force generation test, which is a qualitative test. Saccadic velocity assessment may be the best way to confirm the palsy. Suggested laboratory saccadic velocity tests are electro-oculograghy, infrared scleral reflection technique, and scleral search coil technique. The latter technique is regarded as the most sensitive method. ${ }^{14}$ Our overcorrected cases may have had some residual LR activity, and if we could use above mentioned techniques, some of these cases may be excluded as having complete sixth nerve palsy. Because of limited cost and availability, they have not been widely used and this was applied to our patients. In 1997, Foster ${ }^{2}$ reported that augmentation of VRT significantly increased tonic abducting force of the transposition without decreasing adduction. In a few patients, stiffness of MR decreased; and in most patients, MR weakening was not needed. According to our results, we suggest that in preoperation deviation of $<45$, it is reasonable to perform augmented VRT without BTX to MR. We observed limitation of adduction in 5 patients $(-1$ to -2$)$, and 3 of these patients were among overcorrected cases. In all 4 patients with consecutive $\mathrm{XT}$, unilateral or bilateral MR resection was performed, which leads to orthotropia and improvement of adduction deficit. We did not observe anterior segment ischemia in these patients.

We also compared the results of congenital and traumatic patients. The results are comparable without any statistically significant difference between the two groups.

One of the most important and challenging complications of VRT is the undesired vertical deviation, which has been reported between $5 \%$ and $30 \%$ of the time. Four of our patients had hypotropia ranging from 8 to $30 \mathrm{PD}$.

The reason of this complication is not well understood. Laby and Rosenbaum ${ }^{15}$ believed that differences in tension between nasal and temporal portions of the new insertion causes vertical deviation. Holmes et al ${ }^{16}$ hypothesized that undesirable asymmetry of superior rectus (SR) and inferior rectus (IR) leads to relatively tight IR with resultant hypotropia. Also, anatomical differences between SR and IR may have a role. Some methods suggested to prevent this complication include adjustable suture technique, ${ }^{11}$ intraoperative monitoring of torsion, ${ }^{16}$ and modification of Foster technique using single posterior fixation suture. ${ }^{17}$ Ruth $e t ~ a l^{18}$ performed vertical forced ductions at the end of procedures to ensure there are no vertical restrictions. They managed induced hypotropia by releasing scleral suture and then recessing IR until the forced duction became negative, then posterior fixation suture replaced $8 \mathrm{~mm}$ posterior to LR insertion along the inferior and superior lateral border of IR. A patient with induced hypotropia of $30 \mathrm{PD}$ underwent two consecutive corrective surgeries, according to the method described above, which reduced hypotropia to $10 \mathrm{PD}$. Three other patients with induced 
hypotropia declined corrective surgery. Recently, Holmes et $a l^{16}$ suggested intraoperative monitoring of torsion to reduce the rate of induced vertical deviation after VRT. To the best of our knowledge, our study is the first to report of long-term results of augmented VRT with intraoperative BTX.

\section{Conclusion}

Augmented VRT with intraoperative BTX injection to MR has a high success rate and favorably improves abduction deficit, especially in severe forms of LR palsy.

\section{Summary}

What was known before

- Complete sixth nerve palsy needs transposition of vertical recti toward LR.

- Some advocated injection of Botulinum toxin to MR (intraoperative or postoperation).

\section{What this study adds}

- We performed augmented vertical recti transposition with intraoperative injection of botulinum toxin to MR (to the best of our knowledge there was no published study about this technique).

- The success rate of this technique is promising, especially in improving abduction deficiency.

- The most important disadvantages of this technique are induced hypotropia, and overcorrection in moderate angle deviation.

\section{Conflict of interest}

The authors declare no conflict of interest.

\section{References}

1 O'Connor R. Tendon transplantation in ocular muscle paralysis. Am J Ophthamol 1935; 18: 813-820.

2 Foster RS. Vertical muscle transposition augmented with lateral fixation. J AAPOS 1997; 1: 20-30.
3 Saunders RA, Phillips MS. Anterior segment ischemia after three rectus muscle surgery. Ophthalmology 1988; 95: 533-537.

4 Rosenbaum AL, Kushner BJ, Kirschen D. Vertical rectus transposition and botulinum toxin (oculinum) to medial rectus for abducens palsy. Arch Ophthalmol 1989; 107: 820-823.

5 Fitzsmons R, Lee JP, Elston J. Treatment of sixth nerve palsy in adults with combined botulinum toxin chemodenervation and surgery. Ophthalmology 1988; 95: 1535-1542.

6 Flanders M, Qahtani F, Gans M, Beneish R. Vertical rectus muscle transposition and botulinum toxin for complete sixth nerve palsy. Can J Ophthalmol 2001; 36: 18-25.

7 Armenia JV, Sigal MB. Abducens paralysis repaired with muscle transposition and intraoperative botulinum toxin. Ann Ophthalmol 1987; 19: 416-618, 422.

8 Scott AB. Botulinum injection of eye muscle to correct strabismus. Trans Am Ophthalmol Soc 1981; 79: 734-770.

9 Scott AB, Kraft SP. Botulinum toxin injection in the management of lateral rectus paresis. Ophthalmology 1985; 92: 676-683.

10 Schillinder RJ. A New type of tendon transplant operation for abducens paralysis. J Int Coll Surg 1959; 31: 593-600.

11 Phamanvaechavan P, Anwar D, Guyton D. Adjustable suture technique for enhanced transposition surgery for extra ocular muscles. J AAPOS 2010; 14: 399-405.

12 Leiba H, Wirth GM, Amstuz C, Landau K. Long-term result of vertical transposition and botulinum toxin for sixth nerve palsy. J AAPOS 2010; 14: 498-501.

13 Bansal S, Khan J, Marsh IB. Unaugmented vertical muscle transposition surgery for chronic sixth nerve paralysis. Strabismus 2006; 14: 177-181.

14 Santiago AP, Rosenbaum AL. Clinical Strabismus Management, 1st ed. Saunders: Philadelphia, PA, USA, 1999.

15 Laby DM, Rosenbaum AL. Adjustable vertical rectus muscle transposition surgery. J Pediatr Ophthalmol Strabismus 1994; 31: 75-78.

16 Holmes JM, Hatt SR, Leske DA. Intraoperative monitoring of torsion to prevent vertical deviations during augmented vertical rectus transposition surgery. J AAPOS 2012; 16: 136-140.

17 Struck MC. Augmented vertical rectus transposition surgery with single posterior fixation suture: Modification of foster technique. J AAPOS 2009; 13: 343-349.

18 Ruth AL, Velez FG, Rosenbaum AL. Management of vertical deviation after vertical rectus transposition surgery. J AAPOS 2009; 13: 16-19. 